\title{
Prostate Adenocarcinoma Metastasis and Papillary Thyroid Carcinoma: A Case Report of Coexisting Thyroid Tumors
}

\author{
John Young ${ }^{\mathrm{a}}$, Lindsay Potdevin ${ }^{\mathrm{a}, \mathrm{b}}$, Tomer Davidov ${ }^{\mathrm{a}}$, Arash Mohebati \\ Michael Nagara ${ }^{\text {a }}$ Stanley Trooskin ${ }^{\mathrm{a}}$
}

\begin{abstract}
Metastasis to the thyroid is a relatively unusual clinical entity with prostatic carcinoma metastasizing to the thyroid rarely reported in the literature. We present a case of an 87 year old male who underwent total thyroidectomy for a $2 \mathrm{~cm}$ papillary thyroid carcinoma with a second neighboring hypercellular focus suggestive of follicular variant papillary carcinoma. Immunohistochemical staining was negative for thyroglobulin and positive for PSA, indicating a metastatic focus of prostate cancer. We report a case of a patient with prostate cancer metastasis to the thyroid near a coexisting papillary thyroid carcinoma.
\end{abstract}

Keywords: Metastasis to thyroid; Prostate cancer metastasis; Thyroid cancer

\section{Introduction}

Metastatic disease to the thyroid gland is a rare clinical entity; however, it seems to be more common than expected based on autopsy reports and case presentations $[1,2]$. When this occurs, the most common primary tumor sites are the lungs, breast, kidney, gastrointestinal tract, uterus, skin, lymphoid tissue, and the head and neck [1-6].

In the United States, prostate cancer is the most common of all newly diagnosed cancers and is the second leading cause of cancer death among males, despite recent downward trends in mortality $[7,8]$. Prostate cancer most frequently metastasizes hematogenously to the bone, lung,

Manuscript accepted for publication June 6, 2012

${ }^{\mathrm{a}}$ Department of Surgery, Robert Wood Johnson Medical School, MEB 596, P.O. Box 19, New Brunswick, NJ 08903-0019, USA

${ }^{\mathrm{b}}$ Corresponding author: Lindsay Potdevin. Email: potdevlb@umdnj.edu

doi: http://dx.doi.org/10.4021/jcs77w liver, pleura, and adrenals [9]. Prostate cancer metastasizing to the thyroid has been rarely reported in the literature [10-13].

A "collision tumor" refers to two coexistent but histologically distinct malignant tumors in the same organ, often resulting in an environment favorable for cellular intermingling and tumor growth. Collision tumors in the thyroid have been reported, typically involving papillary and medullary carcinoma or papillary and follicular carcinoma [14]. Collision and coexistent tumors involving metastasis to the thyroid have been rarely reported [14-17], none involving prostate and thyroid carcinoma.

Here, we present a case of a prostate cancer metastasis and a papillary thyroid carcinoma coexistant in the same thyroid lobe.

\section{Case Report}

\section{Patient}

An 87 year old man was referred to our department for management of a $4.2 \mathrm{~cm}$ thyroid nodule which on fine needle aspiration biopsy was positive for papillary thyroid carcinoma. He had initially presented to his primary care physician with a chief complaint of 20 pound weight loss in the past 6 months. A thyroid mass was palpated on physical examination. The patient had denied any voice changes, heat or cold intolerance, history of radiation exposure, and family history of thyroid cancer. The patient did have nocturia, frequency, and decreased force of stream, as well as elevated PSA, but had not yet had a prostate biopsy. Physical exam revealed a firm mass in the left side of the neck with tracheal deviation. TSH level was within normal limits at 1.6. A CT scan revealed a $4.2 \times 3.9 \mathrm{~cm}$ mass with central necrosis effacing the airway, compatible with a substernal thyroid mass. CT scan of the pelvis revealed prostatic calcification, but no significant enlargement. There was also a sclerotic focus in the left ilium and irregular osseous densities in the left acetabular and supra-acetabular regions that were suspicious for metastatic deposits or possibly Paget's disease. A thyroid uptake and scan was performed that yielded 4 and 24 hour uptake 
values of $3 \%$ which were below the normal level. Fine needle aspiration of the thyroid showed nuclear grooves and intranuclear inclusions, diagnostic of papillary cell carcinoma of the thyroid (Bethesda VI).

A total thyroidectomy was performed. Postoperatively, patient developed urinary retention and on subsequent urologic evaluation, he was found to have an elevated PSA of $268 \mathrm{ng} / \mathrm{mL}$. The patient was discharged home on the second postoperative day.

\section{Pathology}

Final pathology diagnosis from the thyroidectomy identified two nodular structures within the left lobe. The first was a 3 $\mathrm{cm}$ multiloculated cyst that contained a smaller $2 \mathrm{~cm}$ focus of papillary carcinoma (T2). The second nodule measured $5 \mathrm{~cm}$ in maximum diameter and contained a single separate focus of presumed follicular variant papillary carcinoma measuring less than $0.2 \mathrm{~cm}$ in maximum. Extensive lymphocytic thyroiditis was seen. No lymphovascular invasion was identified and the margins of resection were free of tumor. The right lobe also showed lymphocytic thyroiditis, and was negative for tumor.

Given the post operative events and a suspicion of prostate cancer with possible disease to the bone, immunohistochemical stains were subsequently performed on the small follicular appearing lesion. The lesion was thyroglobulin negative, but strongly positive for prostate specific antigen. These immunohistochemical staining properties were consistent with a metastatic focus of prostate carcinoma. The patient was subsequently diagnosed with prostatic adenocarcinoma (Gleason 7 score) through outpatient urologic work up.

\section{Discussion}

Metastases to the thyroid may be a more common occurrence than previously believed [2, 18]. In autopsy series, thyroid metastases rates have ranged from $0.5 \%$ in unselected studies to $24 \%$ in patients with widespread non-thyroid malignant neoplasms [5, 18-20]. In these studies, the primary neoplasms arise most commonly from the breast, lung, lymphoid tissue, and skin and led to clinically occult thyroid metastases [5, 18-20]. Despite these postmortem incident rates, clinically, thyroid metastases remain fairly uncommon and therefore present a diagnostic difficulty [1, 2, 21]. Clinical series of symptomatic thyroid metastases have found that primary carcinomas of the kidney, breast, and lung are the most common [1-3, 21]. Some authors have hypothesized that increases in the number of these cases at an institution may be due to increased case findings or increased screening and fine needle biopsies [1].

Metastatic prostate cancer to the thyroid has rarely been reported. To our knowledge, there have been only 4 cases previously described [10-13]. The diagnosis was aided by the positive prostate specific antigen immunohistochemistry, which has been shown to be highly sensitive and specific for all types of prostatic tissue [22]. In all of these cases, prostate cancer had already been identified at least one year prior to the diagnosis of the thyroid metastasis [10-13]. In one of these cases, the prostate cancer metastasized to a thyroid follicular adenoma [10]. In none of these cases was a primary papillary thyroid carcinoma also found [10-13].

Collision tumors involving metastasis to the thyroid have been rarely reported in the literature. These reported collision tumors have been a metastatic liposarcoma and thyroid papillary carcinoma, a follicular variant of papillary carcinoma and squamous carcinoma of unidentified origin, a squamous cell carcinoma of the larynx and thyroid papillary carcinoma, and lung adenocarcinoma and follicular variant of papillary thyroid carcinoma [14-17]. Several hypotheses as to how these collision tumors arise have been put forth. Chance accidental meeting of the two primary tumors, a common carcinogenic stimulus such as radiation within the affected region, and the affects of the first tumor on the microenvironment favoring the development of the second tumor have all been proposed as mechanisms of the development of collision tumors $[14,15]$. To be considered a true collision tumor, the two tumors must have some sort of interface between the two that may or may not allow for some degree of intermingling. However, it is likely that many of these develop as separate entities before one or both of them grow towards each other, thus "colliding".

Our initial preoperative diagnosis was consistent with papillary thyroid carcinoma. Given the size of the substernal thyroid mass, the cold nodule, and the fine-needle aspiration results, we proceeded with total thyroidectomy. On the final pathology in addition to the papillary thyroid carcinoma in the left lobe, a small focus of prostate cancer metastasis was found. This metastatic lesion was diagnosed with a positive PSA and negative thyroglobulin stain. It is uncertain which tumor arose first in the thyroid, but it is certainly possible that the first tumor may have altered the microenvironment increasing the likelihood of the second tumor. While the tumors were found in close proximity to each other, they had not developed into a true collision tumor as there was thyroid tissue separating the two. What is also unusual in this case is that the diagnosis of prostate carcinoma was first made by the finding of metastasis in the thyroid. To our knowledge, this is the first report of coexisting thyroid tumors involving a prostate metastasis and a papillary thyroid carcinoma.

Although thyroid metastasis from a primary adenocarcinoma of the prostate is rare, it can occur as was seen in this patient with a very high PSA at $268 \mathrm{ng} / \mathrm{mL}$. Whether papillary thyroid carcinoma provides a microenvironment conducive to metastatic collision tumors requires further investigation. 


\section{References}

1. Kim TY, Kim WB, Gong G, Hong SJ, Shong YK. Metastasis to the thyroid diagnosed by fine-needle aspiration biopsy. Clin Endocrinol (Oxf). 2005;62(2):236-241.

2. Nakhjavani MK, Gharib H, Goellner JR, van Heerden JA. Metastasis to the thyroid gland. A report of 43 cases. Cancer. 1997;79(3):574-578.

3. Chacho MS, Greenebaum E, Moussouris HF, Schreiber K, Koss LG. Value of aspiration cytology of the thyroid in metastatic disease. Acta Cytol. 1987;31(6):705-712.

4. Cooper David S, G.F.S., Ladenson Paul W, Chapter 8. The Thyroid Gland, in Greenspan's Basic and Clinical Endocrinology, S.D. Gardner DG, Editor.

5. Lam KY, Lo CY. Metastatic tumors of the thyroid gland: a study of 79 cases in Chinese patients. Arch Pathol Lab Med. 1998;122(1):37-41.

6. Chen H, Nicol TL, Udelsman R. Clinically significant, isolated metastatic disease to the thyroid gland. World J Surg. 1999;23(2):177-180; discussion 181.

7. Baade PD, Coory MD, Aitken JF. International trends in prostate-cancer mortality: the decrease is continuing and spreading. Cancer Causes Control. 2004;15(3):237-241.

8. Jemal A, Siegel R, Ward E, Hao Y, Xu J, Murray T, Thun MJ. Cancer statistics, 2008. CA Cancer J Clin. 2008;58(2):71-96.

9. Bubendorf L, Schopfer A, Wagner U, Sauter G, Moch H, Willi N, Gasser TC, et al. Metastatic patterns of prostate cancer: an autopsy study of 1,589 patients. Hum Pathol. 2000;31(5):578-583.

10. Ro JY, Guerrieri C, el-Naggar AK, Ordonez NG, Sorge JG, Ayala AG. Carcinomas metastatic to follicular adenomas of the thyroid gland. Report of two cases. Arch Pathol Lab Med. 1994;118(5):551-556.

11. Selimoglu H, Duran C, Saraydaroglu O, Guclu M, Kiyici S, Ersoy C, Eren MA, et al. Prostate cancer metastasis to thyroid gland. Tumori. 2007;93(3):292-295.
12. Bayram F, Soyuer I, Atmaca H, Demirci D, Gokce C, Canoz O, Unluhizarci K, et al. Prostatic adenocarcinoma metastasis in the thyroid gland. Endocr J. 2004;51(4):445-448.

13. Momma T, Kimura S, Saito S, Onoda N. [Prostate cancer with high serum level of CEA and CA19-9: a case report]. Hinyokika Kiyo. 1998;44(3):187-191.

14. Jacobson AS, Wenig BM, Urken ML. Collision tumor of the thyroid and larynx: a patient with papillary thyroid carcinoma colliding with laryngeal squamous cell carcinoma. Thyroid. 2008;18(12):1325-1328.

15. Brandwein-Gensler M, Urken M, Wang B. Collision tumor of the thyroid: a case report of metastatic liposarcoma plus papillary thyroid carcinoma. Head Neck. 2004;26(7):637-641.

16. Walvekar RR, Kane SV, D'Cruz AK. Collision tumor of the thyroid: follicular variant of papillary carcinoma and squamous carcinoma. World J Surg Oncol. 2006;4:65.

17. Hashimoto K, Yamamoto H, Nakano T, Oyama M, Shiratsuchi $\mathrm{H}, \mathrm{Nakashima} T$, Tamiya $\mathrm{S}$, et al. Tumor-to-tumor metastasis: lung adenocarcinoma metastasizing to a follicular variant of papillary thyroid carcinoma. Pathol Int. 2011;61(7):435-441.

18. Shimaoka K, Sokal JE, Pickren JW. Metastatic neoplasms in the thyroid gland. Pathological and clinical findings. Cancer. 1962;15:557-565.

19. Silverman, S.G.a.V., R.A., Metastatic tumors in the thyroid. Pacific Medical Surgery. 1966;74:175-180.

20. Hull OH. Critical analysis of two hundred twenty-one thyroid glands; study of thyroid glands obtained at necropsy in Colorado. AMA Arch Pathol. 1955;59(3):291311.

21. Ivy HK. Cancer metastatic to the thyroid: a diagnostic problem. Mayo Clin Proc. 1984;59(12):856-859.

22. Oesterling JE. Prostate specific antigen: a critical assessment of the most useful tumor marker for adenocarcinoma of the prostate. J Urol. 1991;145(5):907-923. 